\title{
50 Years of Zweifel Olefination: A Transition-Metal-Free Coupling
}

\author{
Roly J. Armstrong \\ Varinder K. Aggarwal* (D)
}

School of Chemistry, University of Bristol, Cantock's Close, Bristol, BS8 1TS, UK

v.aggarwal@bristol.ac.uk

Dedicated to Professor Herbert Mayr on the occasion of his $70^{\text {th }}$ birthday
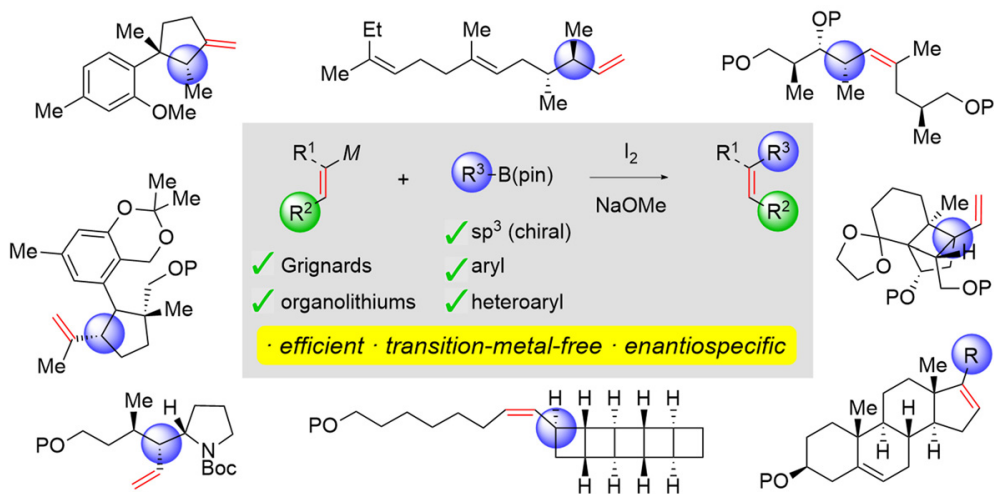

Received: 15.05 .2017
Accepted after revision: 16.05 .2017
Published online: 11.07 .2017
DOI: $10.1055 /$ s-0036-1589046; Art ID: ss-2017-z0328-sr
License terms: CC

Abstract The Zweifel olefination is a powerful method for the stereoselective synthesis of alkenes. The reaction proceeds in the absence of a transition-metal catalyst, instead taking place by iodination of vinyl boronate complexes. Pioneering studies into this reaction were reported in 1967 and this short review summarizes developments in the field over the past 50 years. An account of how the Zweifel olefination was modified to enable the coupling of robust and air-stable boronic esters is presented followed by a summary of current state of the art developments in the field, including stereodivergent olefination and alkynylation. Finally, selected applications of the Zweifel olefination in targetoriented synthesis are reviewed.

1 Introduction

2.1 Zweifel Olefination of Vinyl Boranes

2.2 Zweifel Olefination of Vinyl Borinic Esters

2.3 Extension to Boronic Esters

3.1 Introduction of an Unsubstituted Vinyl Group

3.2 Coupling of $\alpha$-Substituted Vinyl Partners

3.3 Syn Elimination

4 Zweifel Olefination in Natural Product Synthesis

$5 \quad$ Conclusions and Outlook

Key words Zweifel olefination, coupling, boronic esters, alkenes, transition-metal-free, enantiospecific

\section{Introduction}

The stereocontrolled synthesis of alkenes is a topic that has attracted a great deal of attention owing to the prevalence of this motif in natural products, pharmaceutical agents and materials. ${ }^{1}$ Of the many olefination methods that exist, the Suzuki-Miyaura coupling represents a highly convergent method to assemble alkenes (Scheme 1, a). ${ }^{2}$ However, although the coupling of vinyl halides with primary and $\mathrm{sp}^{2}$ boronates takes place effectively, the coupling

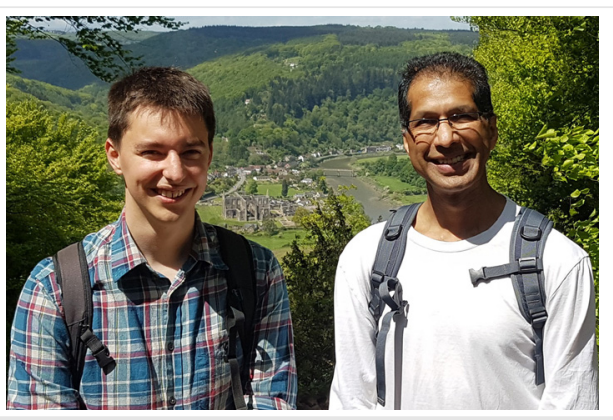

Varinder K. Aggarwal (right) studied chemistry at Cambridge University and received his Ph.D. in 1986 under the guidance of Dr Stuart Warren. After postdoctoral studies (1986-1988) under Professor Gilbert Stork, Columbia University, he returned to the UK as a Lecturer at Bath University. In 1991, he moved to Sheffield University, where he was promoted to Professor in 1997. In 2000, he moved to Bristol University where he holds the Chair in Synthetic Chemistry. He was elected Fellow of the Royal Society in 2012.

Roly J. Armstrong (left) graduated with an MSci in Natural Sciences from Pembroke College, Cambridge (2011) spending his final year working in the laboratory of Professor Steven Ley. He subsequently moved to Merton College, Oxford to carry out a DPhil under the supervision of Professor Martin Smith (2011-2015) working on asymmetric counterion-directed catalysis. In October 2015, he joined the group of Professor Varinder Aggarwal at the University of Bristol as a postdoctoral research associate.

of secondary and tertiary (chiral) boronates remains problematic. ${ }^{3}$ Furthermore, the high cost and toxicity of the palladium complexes required to catalyze these processes also detract from the appeal of this methodology. ${ }^{4}$

The Zweifel olefination represents a powerful alternative to the Suzuki-Miyaura reaction, enabling the coupling of vinyl metals with enantioenriched secondary and tertiary boronic esters with complete enantiospecificity (Scheme $1, \mathrm{~b}) .^{5}$ The reaction is mediated by iodine and base and proceeds with no requirement for a transition-metal catalyst. 
This process is based upon pioneering studies reported in 1967 by Zweifel and co-workers on the iodination of vinyl boranes. This short review summarizes the key contributions made over the last 50 years that have enabled this transformation to evolve into an efficient and atom-economical method for the coupling of boronic esters. Recent contributions to the field are described including the development of Grignard-based vinylation, stereodivergent olefination and alkynylation processes. Finally, selected examples of Zweifel olefination in target-oriented synthesis are reviewed to highlight the utility of this methodology.
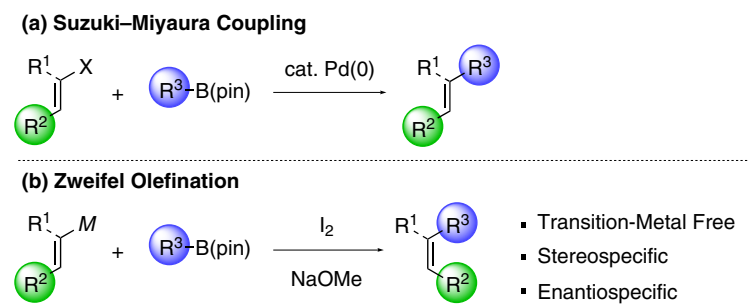

Scheme 1 Olefination of boronic esters

\subsection{Zweifel Olefination of Vinyl Boranes}

In 1967, Zweifel and co-workers reported that vinyl boranes 1, obtained by hydroboration of the corresponding alkynes, could be treated with sodium hydroxide and iodine, resulting in the formation of alkene products 2 (Scheme 2). ${ }^{6}$ Intriguingly, although the intermediate vinyl boranes were formed with high E-selectivity, after addition of iodine, $Z$-alkenes were produced. A reaction with a diastereomerically pure secondary borane afforded the coupled product, 2d, as a single anti diastereoisomer, indicating that the process proceeds with retention of configuration. ${ }^{7}$ Mechanistically, this reaction is thought to proceed by activation of the $\pi$ bond with iodine along with complexation of sodium hydroxide to form a zwitterionic iodonium intermediate 3. This species is poised to undergo a stereospecific 1,2-metalate rearrangement resulting in the formation of a $\beta$-iodoborinic acid 4 . In the presence of sodium hydroxide, this intermediate then undergoes anti elimination to afford the resulting $Z$-alkene product. ${ }^{8}$

Because vinyl borane intermediates could only be accessed by hydroboration of alkynes, the iodine-mediated Zweifel coupling was initially limited to the synthesis of $Z$ alkenes. ${ }^{9}$ However, Zweifel and co-workers subsequently reported an elegant strategy for the complementary synthesis of E-alkenes (Scheme 3). ${ }^{10}$ This transformation was achieved by reacting dialkyl vinyl borane 5 with cyanogen bromide under base-free conditions. Following stereospecific bromination, a boranecarbonitrile intermediate $\mathbf{8}$, was formed, a species that was sufficiently electrophilic to undergo syn elimination. A variety of boranes underwent this transformation, forming alkenes $\mathbf{6 a - c}$ in high yields and

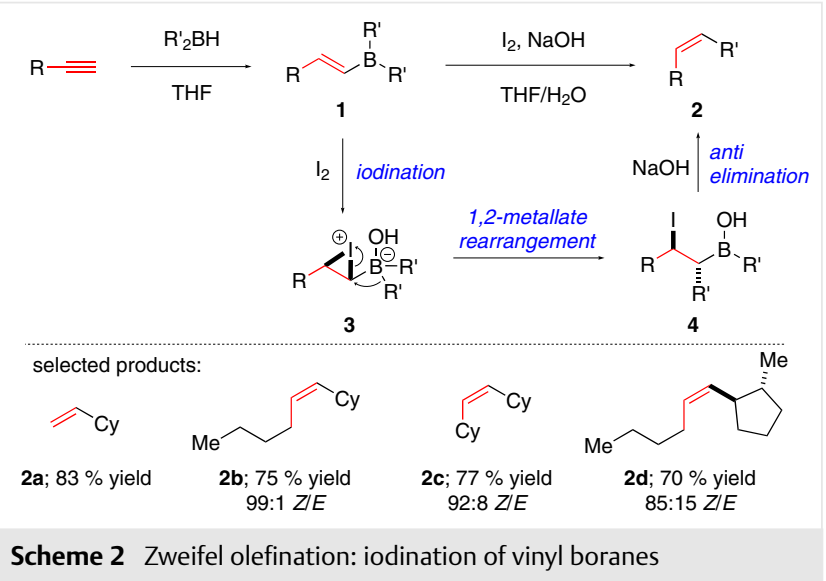

with very high levels of E-selectivity. Chiral non-racemic boranes could be transformed with complete stereospecificity.

$$
\begin{gathered}
\text { THF } \\
\text { Scheme } 3 \text { Synthesis of } E \text {-alkenes using cyanogen bromide } \\
\text { 6a; } 69 \% \text { yield } \\
96: 4 \text { E/Z }
\end{gathered}
$$

A related syn elimination process was reported by Levy and co-workers (Scheme 4). ${ }^{11}$ In this case, a vinyl lithium reagent was prepared by lithium-halogen exchange and then combined with a symmetrical trialkylborane resulting in formation of boronate complex $\mathbf{9}$. Treatment of this intermediate with iodine resulted in stereospecific iodination to produce $\beta$-iodoborane $\mathbf{1 0}$. The enhanced electrophilicity of this species (compared to $\beta$-iodoborinic acids such as $\mathbf{4}$ ) enabled a syn elimination to occur, generating the corresponding trisubstituted alkene $\mathbf{1 1}$ with high levels of stereocontrol. Although the substrate scope of the process is wide, the method was limited to the use of symmetrical trialkyl boranes.

Brown and co-workers demonstrated that the Zweifel olefination can also be applied to the synthesis of alkynes (Scheme 5). ${ }^{12}$ In this case, monosubstituted alkynes were deprotonated to form lithium acetylides, which were reacted with trialkylboranes to form alkynylboronate complexes 12. Addition of iodine triggered a 1,2-metallate rearrangement to generate $\beta$-iodoboranes 13 , which spontaneously 


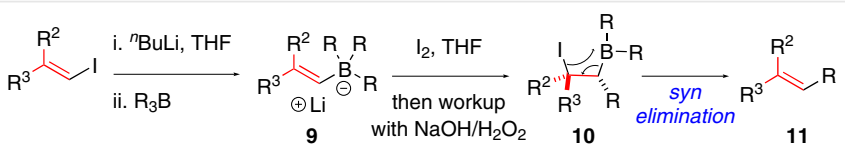

selected products:

Scheme 4 Olefination of symmetrical trialkylboranes

underwent elimination to form alkyne products. This process represents a convenient alternative to the alkylation of lithium acetylides with alkyl halides and has been successfully employed in total synthesis. ${ }^{13}$

\subsection{Zweifel Olefination of Vinyl Borinic Esters}

The transformations described in the previous section suffer from an inherent limitation in that only one of the alkyl groups present in the borane starting materials is incorporated into the alkene product. This is particularly waste-

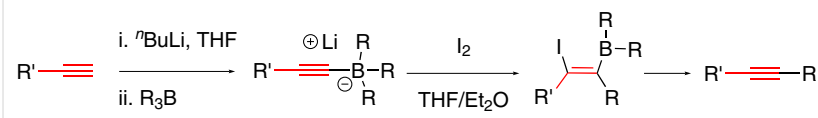

$$
\begin{aligned}
& 12 \\
& 14 \\
& \begin{array}{c}
\text { selected products: } \\
{ }^{n} \mathrm{Bu} \longrightarrow{ }^{n} \mathrm{Bu}
\end{array} \\
& \text { 14a; } 96 \% \text { yield } \\
& { }^{n} \mathrm{Bu}=\mathrm{Cy} \\
& { }^{n} \mathrm{Bu}=\mathrm{Ph} \\
& \mathrm{Ph}=\mathrm{Ph} \\
& \text { 14b; } 99 \% \text { yield } \\
& \text { 14c; } 98 \% \text { yield } \\
& \text { 14d; } 95 \% \text { yield }
\end{aligned}
$$

ful when the borane is challenging to access or expensive. One solution to this problem would be to employ a mixed borane in which one (or two) of the boron-bound groups demonstrates a low migratory aptitude (e.g., thexyl). ${ }^{14}$ However, in practice, determining which group will migrate has proved to be non-trivial and highly substrate-dependent. For example, Zweifel and co-workers showed that treatment of divinylalkylborane $\mathbf{1 5}$ (obtained by double hydroboration of 1 -hexyne with thexylborane) with iodine resulted in competitive migration of both the $\mathrm{sp}^{2}$ and thexyl groups leading to a mixture of the desired product $\mathbf{1 6}$ along with 17 (Scheme 6). ${ }^{15}$ They overcame this problem by treating the intermediate divinylalkylborane $\mathbf{1 5}$ with trimethylamine oxide, resulting in selective oxidation of the $B-C_{\text {thexyl }}$ bond to afford borinic ester 18. Due to the low migratory aptitude of an alkoxy ligand on boron, ${ }^{16}$ addition of iodine and sodium hydroxide now led to selective formation of Z,E-diene 16. Although this allowed control over which group migrated, the method was limited to the synthesis of symmetrical dienes.
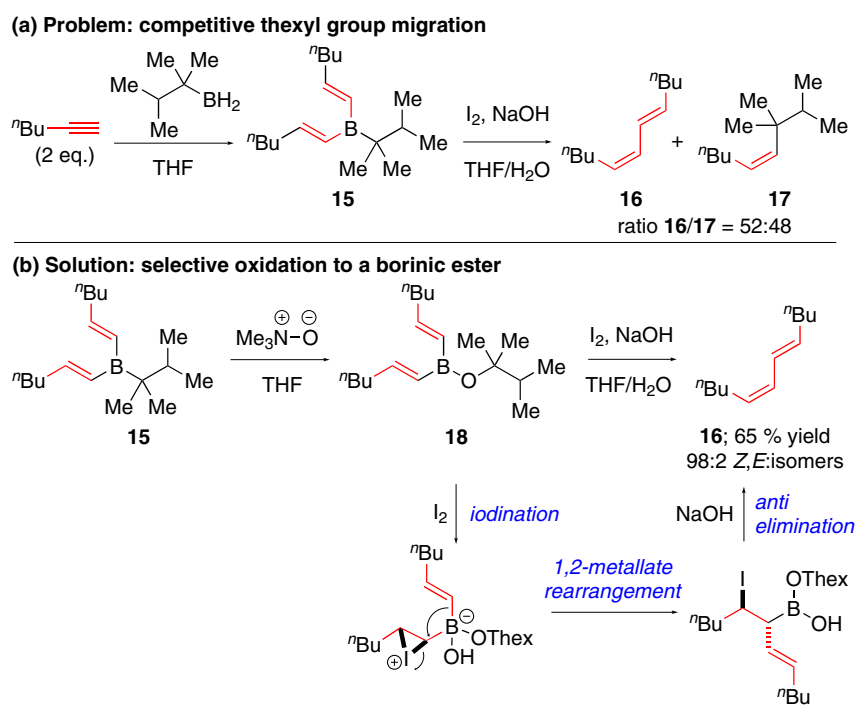

Scheme 6 Diene synthesis by Zweifel olefination of boranes or borinic esters 


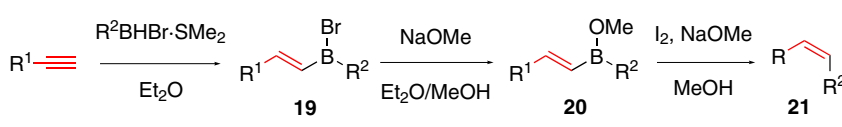

$$
\begin{aligned}
& \text { selected products: } \\
& \text { 21a; } 74 \% \text { yield } \quad \text { 21b; } 69 \% \text { yield } \\
& \text { >99:1 Z/E } \quad>99: 1 \text { Z/E }
\end{aligned}
$$

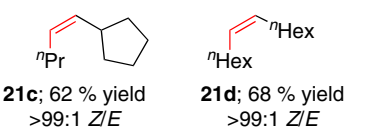

Scheme 7 Synthesis of Z-alkenes from vinyl borinic esters

A more general approach to the iodination of vinyl borinic esters was later reported by Brown and co-workers (Scheme 7) ${ }^{17}$ In this case, non-symmetrical vinyl borinic esters $\mathbf{2 0}$ were obtained by hydroboration of alkynes with alkylbromoboranes followed by methanolysis of the resulting bromoborane intermediates 19. Addition of sodium methoxide and iodine led to alkene products $\mathbf{2 1 a}-\mathbf{d}$ in good yields and very high levels of $Z$-selectivity.

\subsection{Extension to Boronic Esters}

Although the use of borinic esters significantly expanded the potential of the Zweifel olefination, there were still significant problems with this approach, most notably associated with the high air sensitivity of the borane starting materials. In contrast to boranes, boronic esters are air- and moisture-stable materials which can be readily prepared via a wide range of methods. ${ }^{18}$ Evans and Matteson independently recognized the potential of boronic esters as substrates for Zweifel olefination communicating independent studies almost simultaneously..$^{19,20}$

Matteson's coupling process began with the synthesis of a vinyl boronate complex $\mathbf{2 3}$ by addition of an organolithium reagent to a vinyl boronic ester $\mathbf{2 2}$ (Scheme 8). ${ }^{19}$ This intermediate was treated with iodine and sodium hydroxide, resulting in iodination followed by 1,2-metallate rearrangement to form a $\beta$-iodoboronic ester which underwent anti elimination to form the corresponding $Z$-alkene. This reaction could be carried out with alkyl or aryl lithium reagents and the coupled products $\mathbf{2 4 a}$ and $\mathbf{2 4 b}$ were formed in moderate to good yields.

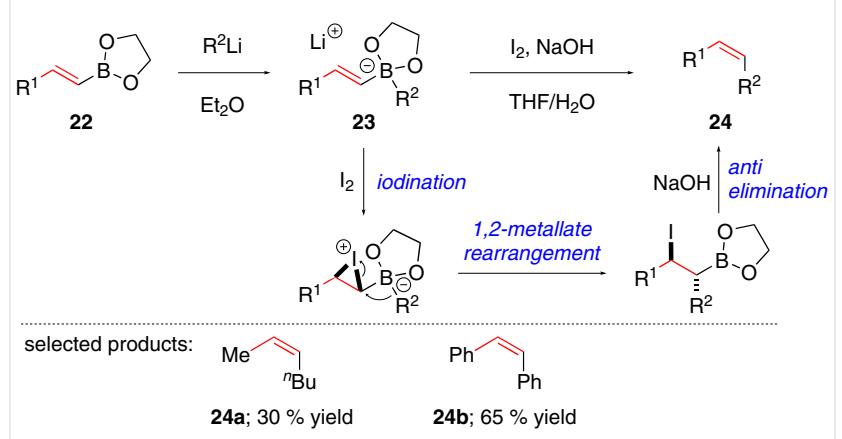

Scheme 8 Zweifel olefination of vinyl boronic esters

Evans and co-workers' strategy also began with formation of a vinyl boronate complex (Scheme 9). ${ }^{20}$ In contrast to Matteson's approach, this intermediate was accessed by reacting $E$-vinyl lithium reagent 26a (prepared by lithiumhalogen exchange) with secondary alkyl boronic ester $\mathbf{2 5}$. Treatment of the resulting vinyl boronate complex 27a with iodine and sodium methoxide resulted in formation of alkene 28a in $75 \%$ yield ( $>96: 4 Z / E$ ). When a $Z$-vinyl lithium precursor 26b was employed, alkene $\mathbf{2 8 b}$ was obtained in $58 \%$ yield with very high E-selectivity. The flexibility derived from the ability to form identical vinyl boronate complexes by either reacting a vinyl boronic ester with an organolithium or a vinyl lithium with a boronic ester is a particularly appealing feature of the Zweifel olefination.

Brown and co-workers subsequently extended this methodology to enable the synthesis of trisubstituted alkenes (Scheme 10). ${ }^{17,21}$ By reacting various trisubstituted

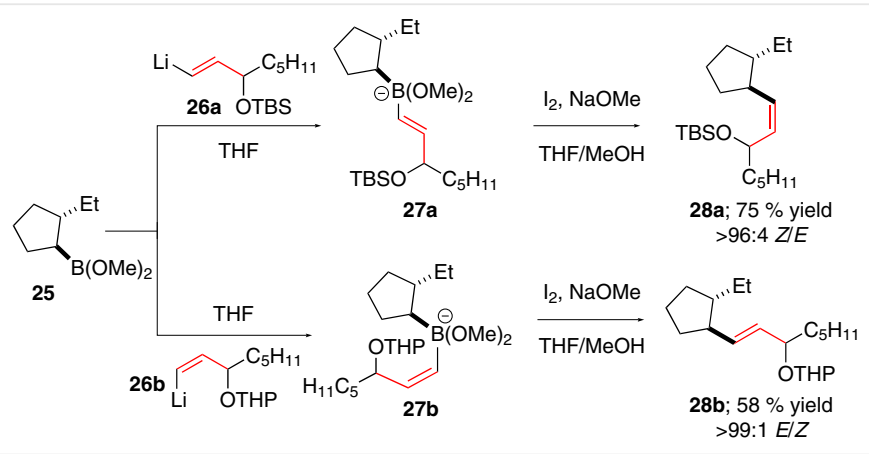

Scheme 9 Zweifel olefination of vinyl lithiums with boronic esters 


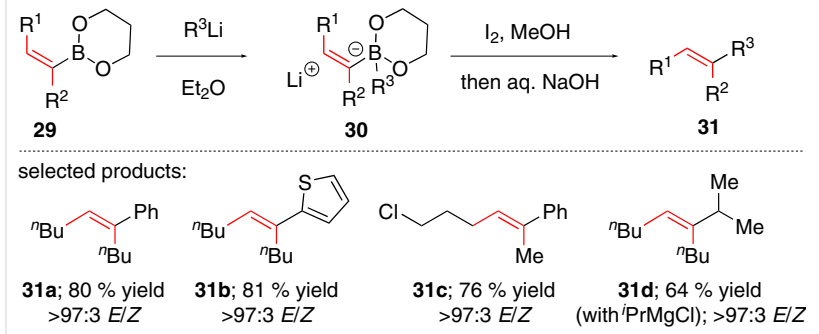

Scheme 10 Zweifel olefination of trisubstituted vinyl boronic esters

vinyl boronic esters (29) with organolithium nucleophiles, a range of products was prepared in good to excellent yields. Notably, heteroaromatic groups could be introduced (in 31b) and alkyl Grignard reagents could be used in place of organolithium reagents (in 31d).

The methods shown in Schemes 8-10 represented a significant advance upon the early work on the Zweifel olefination of boranes and borinic esters. However, at the time the potential of the method was not fully realized owing to the paucity of methods available for the preparation of boronic esters. Consequently, only a handful of studies involving Zweifel olefination were published over the following three decades. ${ }^{22}$ In recent years, the huge increase in methods available for the enantioselective synthesis of boronic esters has led to a renaissance in chemistry based upon the Zweifel olefination. Several new studies into the process have been reported along with elegant reports employing Zweifel olefination in total synthesis. These results are described in the following sections.

\subsection{Introduction of an Unsubstituted Vinyl Group}

The introduction of a vinyl group into a target molecule is commonly required in synthesis owing to the prevalence of this motif in natural products and as a valuable handle for further functionalization. The first report describing the introduction of an unsubstituted vinyl group by Zweifel olefination was published by Aggarwal and co-workers in their stereocontrolled synthesis of (+)-faranal (Scheme 11). ${ }^{23}$ In this process, vinyl lithium was prepared in situ from tetravinyltin by tin-lithium exchange and was then reacted with enantioenriched secondary boronic ester $\mathbf{3 2}$. The resulting vinyl boronate complex was treated with iodine and sodium methoxide, thus promoting 1,2-metallate rearrangement and elimination affording alkene 33. This key intermediate was directly subjected to hydroboration and oxidation to provide alcohol 34 in $69 \%$ yield with very high diastereoselectivity. Oxidation with PCC completed the synthesis of (+)-faranal in $76 \%$ yield.

It was subsequently shown that the vinyl lithium approach could be also be applied to the enantiospecific coupling of trialkyl tertiary boronic esters (Scheme 12, a) ${ }^{24}$ and benzylic tertiary boronic esters ${ }^{25}$ (Scheme $12, \mathrm{~b}$ ). It is noteworthy that in these cases despite the sterically congested nature of the boronic ester starting materials, the coupled products were obtained in excellent yields. The double vinylation of primary-tertiary 1,2-bis(boronic esters) has also been achieved using this approach (Scheme 12, c). ${ }^{26}$ Using four equivalents of vinyl lithium, diene $\mathbf{3 7}$ was obtained in $77 \%$ yield.

$$
\begin{aligned}
& \text { (a) Synthesis of } \alpha \text {-(tertiary trialkyl) alkenes } \\
& \begin{array}{lll}
{ }_{\mathrm{Me}}^{n} \mathrm{Bu} & \text { ii. } \mathrm{I}_{2} \\
& \text { iii. } \mathrm{NaOMe}, \mathrm{Et}_{2} \mathrm{O} / \mathrm{THF} / \mathrm{MeOH} & \begin{array}{c}
35 ; 72 \% \text { yield } \\
100 \% \text { e.s. }
\end{array}
\end{array}
\end{aligned}
$$

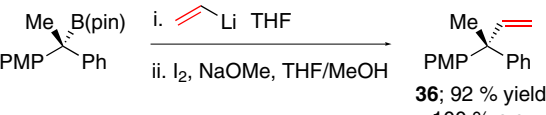

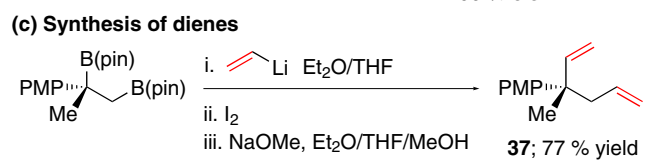

Scheme 12 Applications of Zweifel olefination with vinyl lithium (prepared from tetravinyltin); PMP = p-methoxyphenyl; e.s. = enantiospecificity

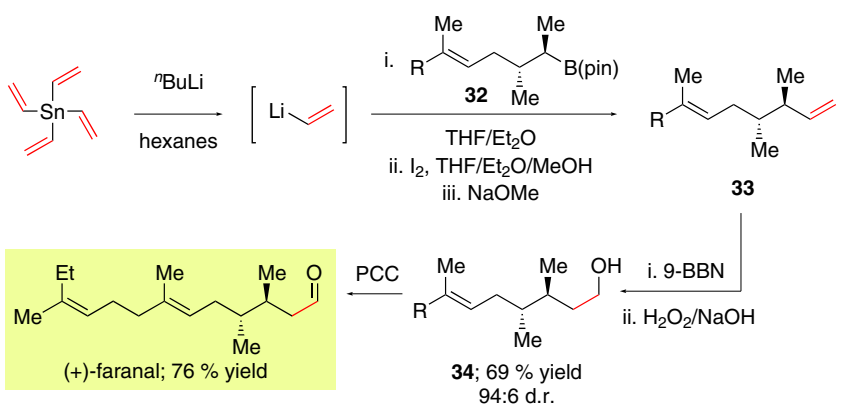

Scheme 11 Introduction of an unsubstituted vinyl group with vinyl lithium: stereoselective synthesis of $(+)$-faranal; $\mathrm{R}=\left(\mathrm{CH}_{2}\right)_{2} \mathrm{CHCMeEt}$; pin $=$ pinacolato 
Vinylation under Zweifel conditions represents a powerful strategy for the synthesis of alkenes. However, the necessity of preparing vinyl lithium in situ from the corresponding toxic stannane or volatile vinyl bromide detracts from the appeal of the process. In contrast, stable THF solutions of vinylmagnesium chloride or bromide are commercially available. ${ }^{27}$ Aggarwal and co-workers have studied the Zweifel olefination of tertiary boronic ester $\mathbf{3 8}$ with vinylmagnesium bromide in $\mathrm{THF}^{25}$ Monitoring the reaction by ${ }^{11} \mathrm{~B}$ NMR spectroscopy revealed that with one equivalent of vinylmagnesium bromide, the expected vinyl boronate complex 39 was not observed and instead a mixture of unreacted boronic ester $\mathbf{3 8}$ and trivinyl boronate complex $\mathbf{4 0}$ was formed (Scheme 13). The latter species originates from over-addition of vinylmagnesium bromide promoted by the high Lewis acidity of the $\mathrm{Mg}^{2+}$ counterion. Upon addition of an excess of vinylmagnesium bromide (4 eq.), trivinyl boronate complex $\mathbf{4 0}$ was obtained exclusively, and after addition of $\mathrm{I}_{2}$ followed by NaOMe, the coupled product 41a was obtained in good yield. These conditions were successfully applied to the synthesis of a series of benzylic tertiary substrates $\mathbf{4 1 a - d}$. The reaction is ineffective at forming very hindered alkenes such as $\mathbf{3 6}$, although this product could be synthesized efficiently with vinyl lithium.

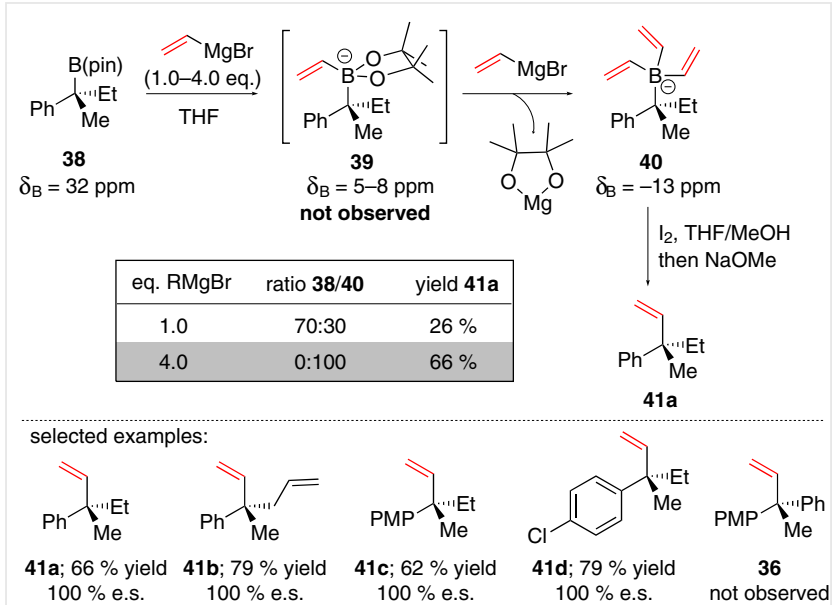

Scheme 13 Zweifel olefination of tertiary boronic esters with vinylmagnesium bromide in THF

Very recently, an improved procedure for coupling unhindered boronic esters with vinylmagnesium chloride has been reported (Scheme 14). ${ }^{28}$ As with tertiary boronic esters, it was observed that addition of vinylmagnesium chloride to a THF solution of secondary boronic ester 42 resulted in over-addition to form trivinyl boronate complex 44. However, if the reaction was carried out in a 1:1 THF/DMSO mixture, ${ }^{29}$ over-addition was completely suppressed and only mono-vinyl boronate complex 43 was obtained. After addition of iodine and sodium methoxide, the coupled product 45a was obtained in $89 \%$ yield. This process pro-

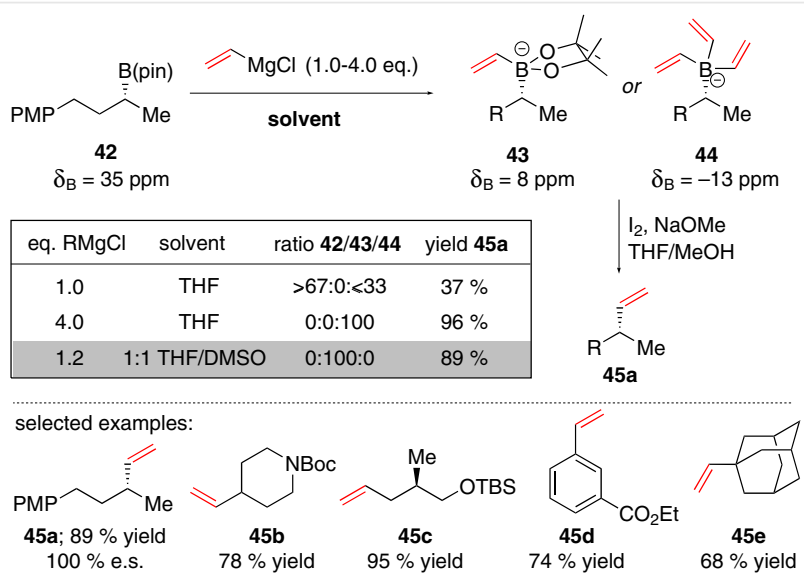

Scheme 14 Zweifel olefination of boronic esters with vinylmagnesium chloride in THF/DMSO; $\mathrm{R}=\left(\mathrm{CH}_{2}\right)_{2} \mathrm{PMP}$

ceeds effectively with a range of primary, secondary and aromatic boronic esters. Notably, the use of the mild Grignard reagent allows chemoselective coupling to occur in the presence of reactive functional groups such as carbamates (in 45b) and ethyl esters (in 45d). Although good yields of product were obtained with unhindered tertiary boronic esters (in 45e), in general, the Zweifel vinylation of tertiary boronic esters is best achieved either with four equivalents of vinylmagnesium halide in THF or with vinyl lithium.

In summary, there are currently three methods available to introduce an unsubstituted vinyl group by Zweifel olefination (Scheme 15). For aromatic, primary and unhindered secondary boronic esters, the desired boronate complex can be formed efficiently using 1.2 equivalents of vinylmagnesium halide in 1:1 THF/DMSO. For the majority of tertiary boronic esters it is recommended to employ four equivalents of vinylmagnesium halide in THF (to form the trivinyl boronate complex), although with extremely hindered tertiary boronic esters, the best results are obtained with vinyl lithium.

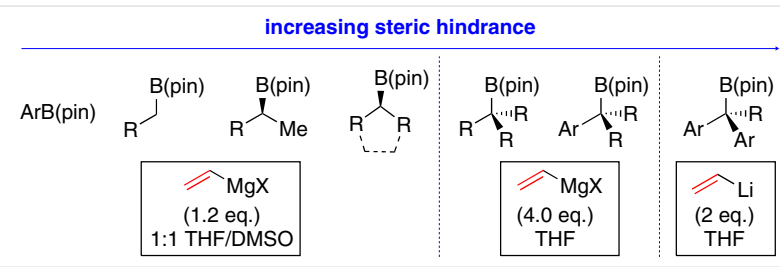

Scheme 15 Summary of the best methods for boronate complex formation for the Zweifel vinylation of various boronic esters; $R$ = alkyl group

\subsection{Coupling of $\alpha$-Substituted Vinyl Partners}

In addition to the synthesis of alkyl-substituted alkenes, the Zweifel olefination has also been applied to the cou- 
pling of vinyl partners $\alpha$-substituted with a heteroatom. The coupling of lithiated ethyl vinyl ether $\mathbf{4 6}$ (readily prepared by deprotonation of ethoxyethene with ${ }^{t} \mathrm{BuLi}$ ) with a tertiary boronic ester proceeded smoothly to provide enol ether 47, which was hydrolyzed under mild conditions to form 48 (Scheme 16, a). ${ }^{25,30}$ This process represents a novel method for the conversion of boronic esters into ketones. This methodology has also been extended to the enantiospecific synthesis of vinyl sulfides (Scheme 16, b). ${ }^{28}$

A related strategy for the alkynylation of boronic esters has recently been reported by Aggarwal and co-workers (Scheme 17). ${ }^{31}$ In contrast to the successful alkynylation reactions of trialkyl boranes discussed previously (Scheme $5),{ }^{12,13}$ boronic esters undergo reversible boronate complex formation with lithium acetylides. This means that addition of electrophiles does not result in coupling, but instead leads to direct trapping of the acetylide and recovery of the boronic ester. A solution to this problem was developed in which vinyl bromides or carbamates were lithiated at the $\alpha$-position with LDA and then reacted with boronic esters in a Zweifel olefination. Treatment of the resulting vinyl bromides or carbamates with base (TBAF for bromides and ${ }^{t} \mathrm{BuLi}$ or LDA for carbamates) triggered elimination to form the corresponding alkynes 50. Coupling of a wide range of secondary and tertiary boronic esters was achieved in excellent yields with complete enantiospecificity.

In 2014, an interesting intramolecular variant of the Zweifel olefination for the construction of four-membered ring products was reported (Scheme 18). ${ }^{32}$ In this process, 51, which possesses both a boronic ester and a vinyl bromide, was treated with tert-butyllithium resulting in chemoselective lithium-halogen exchange followed by spontaneous cyclization to form cyclic vinyl boronate complex 52. Upon treatment with iodine and methanol this species underwent stereospecific ring contraction to provide $\beta$-iodoboronic ester 53. Elimination of this intermediate gave exocyclic alkene $\mathbf{5 4}$ in $63 \%$ yield. It is particularly noteworthy that this challenging Zweifel olefination occurs in good yield despite the highly strained nature of the exomethylene cyclobutene product.

\subsection{Syn Elimination}

Aggarwal and co-workers have reported a method for the synthesis of allylsilanes through a lithiationborylation-Zweifel olefination strategy (Scheme 19). ${ }^{33}$ In this process, silaboronate $\mathbf{5 6}$ was homologated with configurationally stable lithium carbenoids $\mathbf{5 5}$ to provide $\alpha$-silyl-

(a) Synthesis of ketones from boronic esters

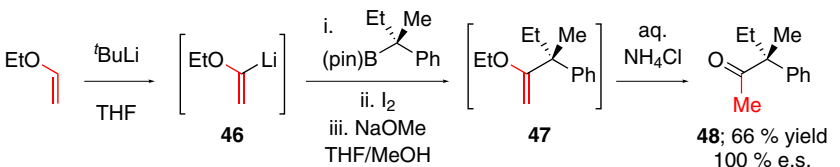

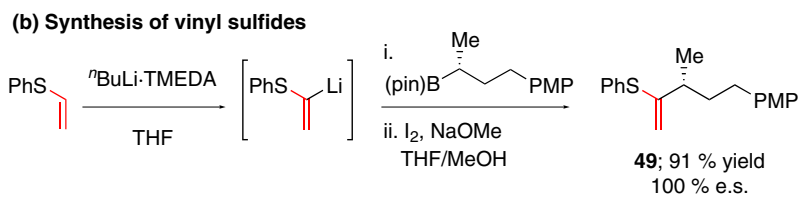

Scheme 16 Synthesis of ketones and vinyl sulfides by Zweifel olefination

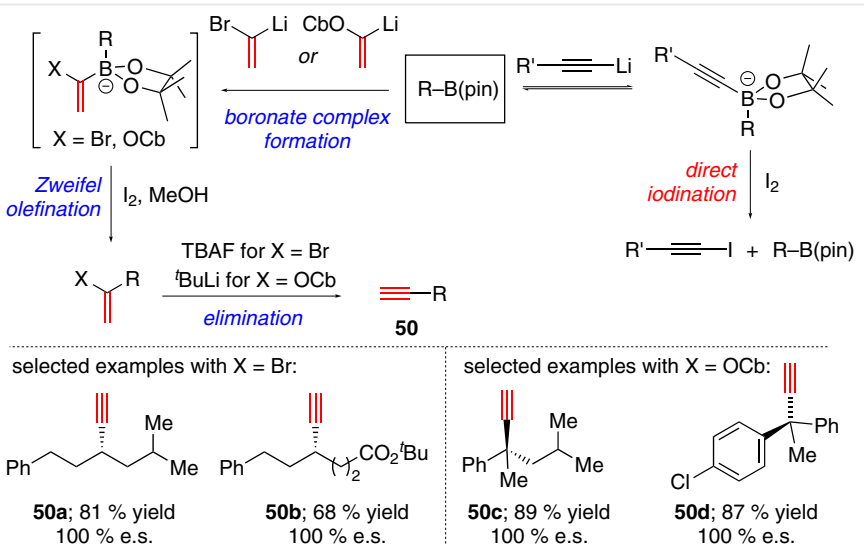

Scheme 17 Alkynylation of enantioenriched boronic esters; $\mathrm{Cb}=\mathrm{C}(\mathrm{O}) \mathrm{N}^{\mathrm{i}} \mathrm{Pr}_{2}$ 
boronic esters 57, which were then subjected to Zweifel olefination to obtain allylsilane products $\mathbf{5 8}$. Notably, it was necessary to carry out the Zweifel olefination without sodium methoxide owing to the instability of the allylsilane products under basic conditions. The substrate scope of the process was wide and a range of allylsilanes was prepared in high yields and with excellent levels of enantioselectivity. Interestingly, with a hindered $\alpha$-silylboronic ester, $E$-crotylsilane 58d was obtained as a single geometrical isomer, but $Z$-crotylsilane 58c was formed in slightly reduced selectivity $(95: 5 \mathrm{Z} / E)$.

To rationalize the reduced selectivity observed in the formation of $Z$-crotylsilane $\mathbf{5 8 c}$, it was postulated that as the boronic ester becomes more hindered, the transition state for anti elimination becomes disfavored due to a steric clash between the bulky $\mathrm{R}^{1}$ and $\mathrm{R}^{2}$ substituents (Scheme 20). This allows the usually less favorable syn elimination pathway to compete, resulting in the formation of small amounts of the $E$-isomer.

Similar behavior has been observed in the Zweifel olefination of hindered secondary boronic esters with alkenyllithiums (Scheme 21) ${ }^{34}$ As the boronic ester becomes more sterically encumbered (for example, benzylic or $\beta$ -

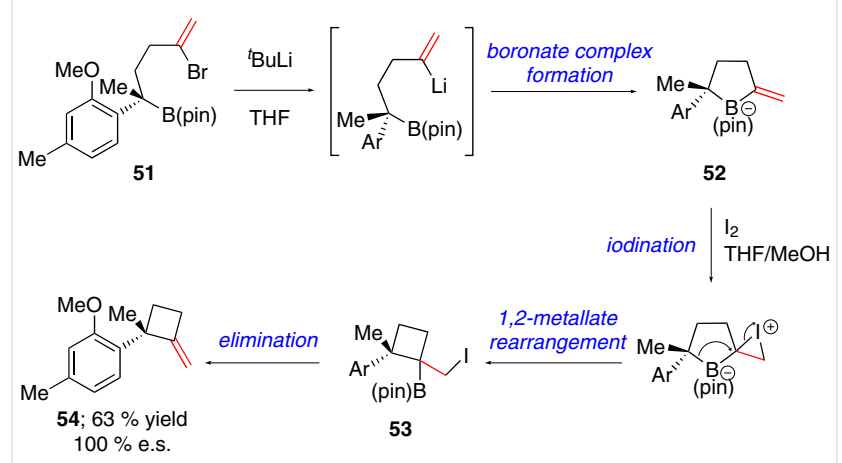

Scheme 18 Construction of an exomethylene cyclobutene by an intramolecular Zweifel olefination; $\mathrm{Ar}=2-\mathrm{MeO}-4-\mathrm{MeC}_{6} \mathrm{H}_{3}$

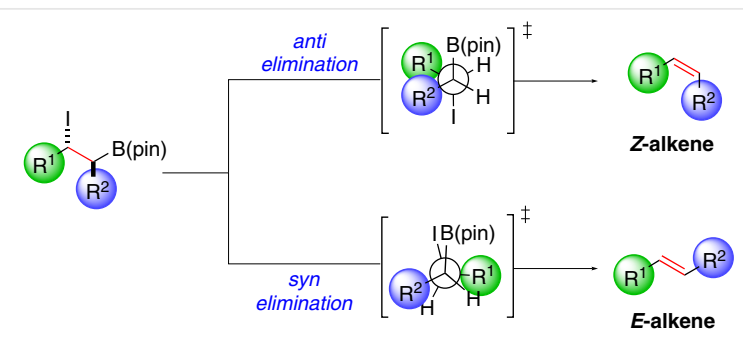

Scheme 20 Rationalization for reduced Z|E selectivity with bulky boronic esters

branched), increasing formation of the $E$-isomer was observed, up to $90: 10 Z / E$ in the case of menthol-derived alkene 60c.
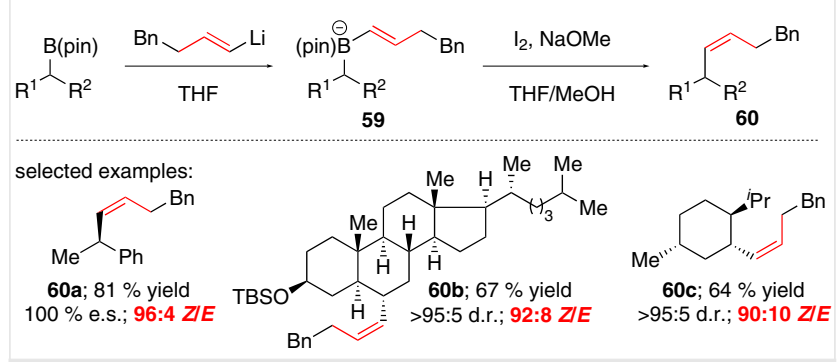

Scheme 21 Reduced Z/E selectivity with bulky boronic esters

In these cases, Aggarwal and co-workers have shown that iodine can be replaced with $\mathrm{PhSeCl}$ resulting in the formation of $\beta$-selenoboronic esters (Scheme 22). ${ }^{35}$ Because the selenide is a poorer leaving group than the corresponding iodide, treatment of these intermediates with sodium methoxide led exclusively to anti elimination providing the coupled products $\mathbf{6 0} \mathbf{a}-\mathbf{c}$ as a single $Z$-isomer in all cases. ${ }^{34}$

It was also demonstrated that $\beta$-selenoboronic esters (obtained by selenation of vinyl boronate complexes) could be directly treated with $m$-CPBA resulting in chemoselective oxidation of the selenide to give the corresponding selenoxide (Scheme 23, a). ${ }^{34}$ A novel syn elimination then occurred in which the selenoxide oxygen atom attacked a boron atom instead of a hydrogen atom, providing $E$ -

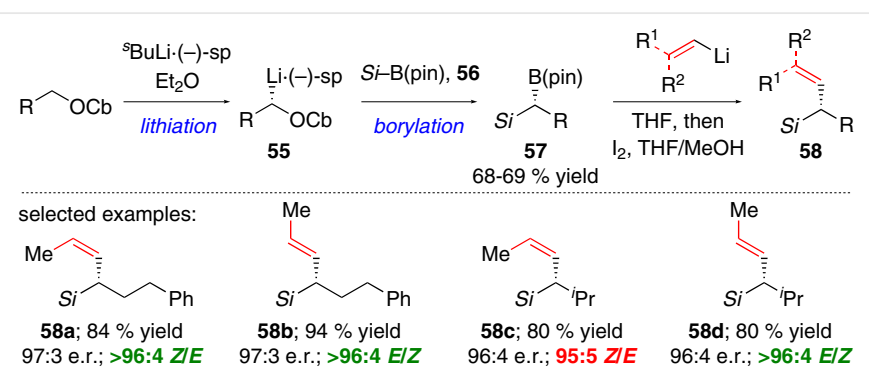

Scheme 19 Synthesis of allyl- and crotylsilanes via a lithiation-borylation-Zweifel olefination strategy; $\mathrm{Si}=\mathrm{SiPhMe}_{2} ;(-)$-sp $=(-)$-sparteine 

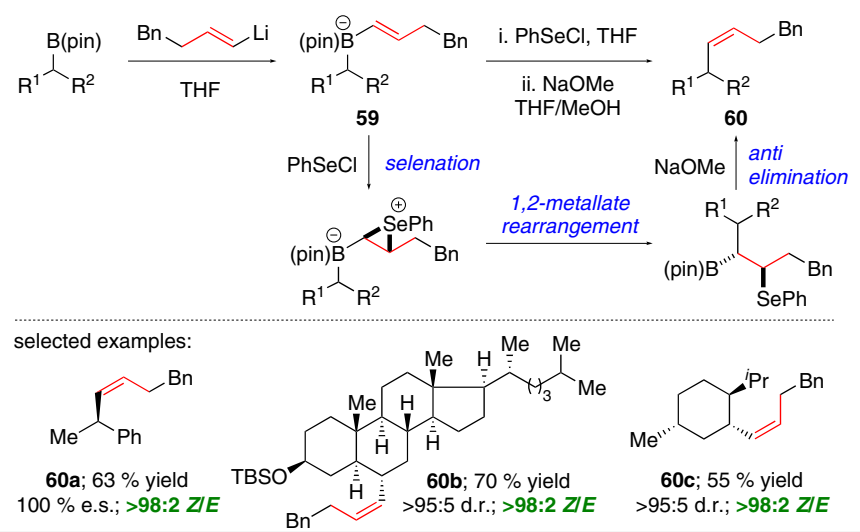

Scheme 22 Highly Z-selective olefination of sterically hindered boronic esters

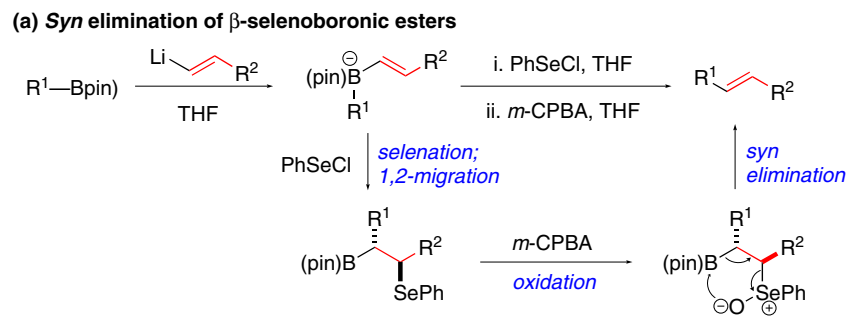

(b) Stereodivergent olefination
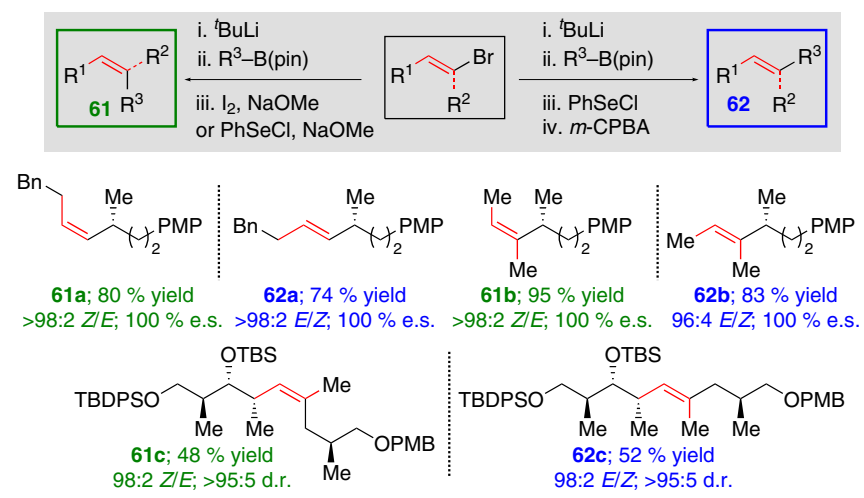

Scheme 23 Stereodivergent olefination of boronic esters

alkenes with high selectivity. In conjunction with the Zweifel olefination (or its $\mathrm{PhSeCl}$-mediated analogue) this represents a stereodivergent method where either isomer of a coupled product can be obtained from a single isomer of vinyl bromide starting material (Scheme 23, b). The substrate scope of both processes is broad and a range of diand trisubstituted alkenes was prepared including 61c which represents the $\mathrm{C} 9-\mathrm{C} 17$ fragment of the natural product discodermolide.

In some cases, the ability to carry out syn elimination of $\beta$-iodoboronic esters is also desirable. For example, very recently Aggarwal and co-workers reported a coupling of cy- clic vinyl lithium reagents with boronic esters (Scheme $24) .{ }^{28}$ In this case, the cyclic $\beta$-iodoboronic ester intermediates 63 cannot undergo bond rotation and therefore must undergo a challenging syn elimination. It was found that this elimination could be promoted by adding an excess of sodium methoxide (up to 20 eq.). Using this methodology, a range of five- and six-membered cycloalkene products 64 were prepared in high yields and with complete stereospecificity, including glycal 64b and abiraterone derivatives such as $\mathbf{6 4 c}$. 
Since the pioneering studies on Zweifel olefination reported by Evans and Matteson, the method has been significantly developed such that a wide range of functionalized alkene products can now be obtained. The final section of this short review showcases selected examples where Zweifel olefination has been used in complex molecule synthesis. ${ }^{36}$

\section{Zweifel Olefination in Natural Product Synthesis}

Aggarwal and co-workers recently reported an 11-step total synthesis of the alkaloid (-)-stemaphylline employing a tandem lithiation-borylation-Zweifel olefination strategy (Scheme 25). ${ }^{37}$ Pyrrolidine-derived boronic ester 65 was homologated with a lithium carbenoid to afford boronic ester 66 in 58 \% yield and 96:4 d.r. A subsequent Zweifel olefination with vinyl lithium (synthesized in situ from tetravinyltin) gave alkene $\mathbf{6 7}$ in $71 \%$ yield. Notably, these two steps could be combined into a one-pot operation, directly providing 67 in $70 \%$ yield. The alkene was later employed in a ring-closing-metathesis-reduction sequence to form the core 5-7 ring system of (-)-stemaphylline.

A recent formal synthesis of the complex terpenoid natural product solanoeclepin A has been reported by Hiemstra and co-workers (Scheme 26) ${ }^{38}$ A key step in this synthe- sis was the vinylation of the bridgehead tertiary boronic ester in 68. Formation of the trivinyl boronate complex with excess vinylmagnesium bromide in THF followed by addition of iodine and sodium methoxide produced alkene 69, which was employed without purification in a subsequent sequence of oxidative cleavage and Horner-WadsworthEmmons olefination to form $\mathbf{7 0}$ in a yield of $67 \%$ over four steps.

Morken and Blaisdell have reported an elegant stereoselective synthesis of debromohamigeran $\mathrm{E}$ that employs a Zweifel coupling of an $\alpha$-substituted vinyl lithium (Scheme 27). ${ }^{39}$ Cyclopentyl boronic ester 72 was prepared from 1,2bis(boronic ester) 71 in $42 \%$ yield by a highly selective hydroxy-directed Suzuki-Miyaura coupling. This intermediate was then subjected to Zweifel coupling with isopropenyllithium (synthesized by $\mathrm{Li}-\mathrm{Br}$ exchange) to form $\mathbf{7 3}$ in $93 \%$ yield. Completion of the synthesis of debromohamigeran E required four further steps including hydrogenation of the alkene to an isopropyl group.

A short enantioselective total synthesis tatanan A was reported by Aggarwal and co-workers, which employs a stereospecific alkynylation reaction (Scheme 28). ${ }^{40}$ Boronic ester 74 (synthesized by a diastereoselective Matteson homologation) was subjected to Zweifel olefination with lithiated vinyl carbamate. Treatment of the resulting vinyl carbamate $\mathbf{7 5}$ with LDA resulted in elimination to form alkyne

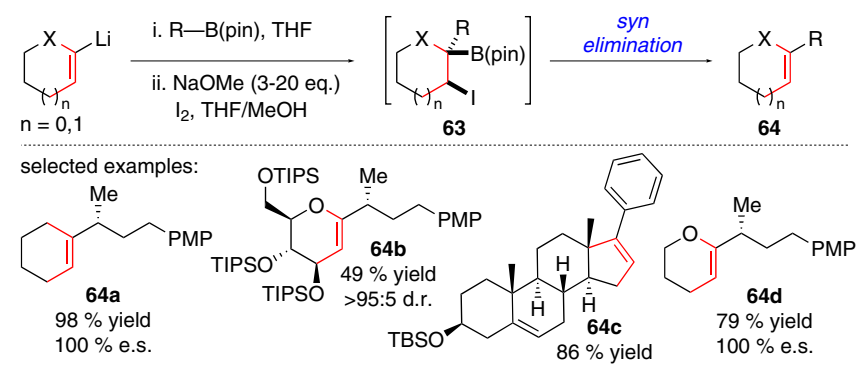

Scheme 24 Synthesis of cycloalkenes via a challenging syn elimination

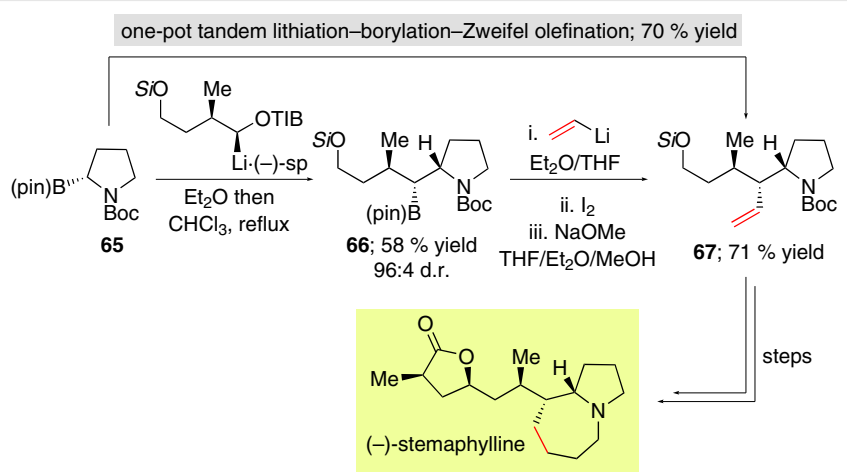

Scheme 25 Stereocontrolled synthesis of (-)-stemaphylline; Si = TBDPS; TIB = 2,4,6-triisoproylbenzoyl 


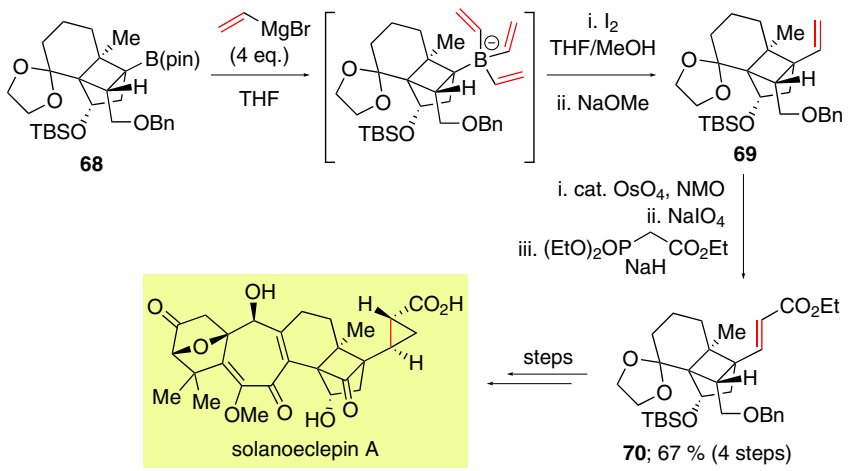

Scheme 26 Formal synthesis of solanoeclepin A
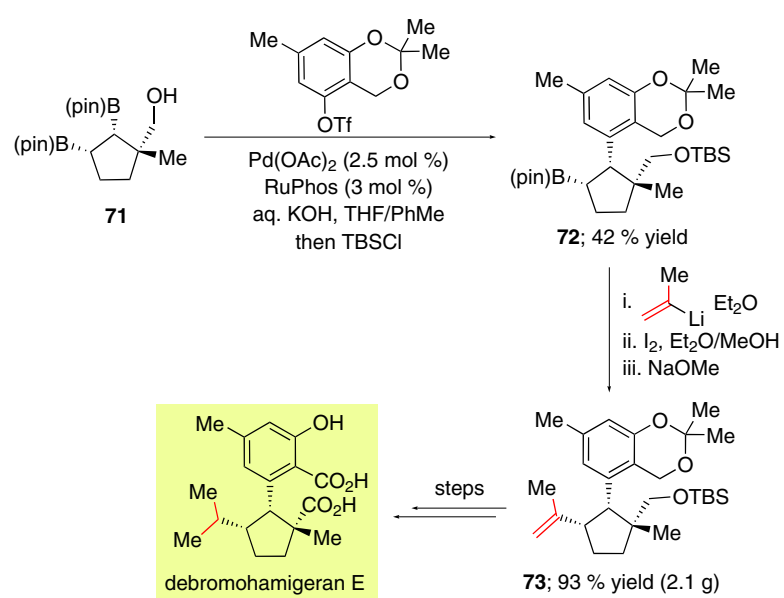

debromohamigeran $\mathrm{E}$

73; $93 \%$ yield $(2.1 \mathrm{~g})$

Scheme 27 Enantioselective synthesis of debromohamigeran E

76 in $97 \%$ yield with complete diastereospecificity. This alkyne was converted into the trisubstituted alkene of tatanan A in two further steps.

A collaborative study on the synthesis of ladderane natural products was recently published by the groups of Boxer, Gonzalez-Martinez and Burns (Scheme 29). ${ }^{41}$ A key intermediate in these studies was the unusual lipid tail [5]-ladderanoic acid. This compound was prepared from mesoalkene $\mathbf{7 7}$ by a sequence involving copper-catalyzed desymmetrizing hydroboration (95\% yield, $90 \%$ ee) followed by Zweifel olefination with vinyl lithium reagent 79 (3:1 $E / Z)$. It was found that carrying out the Zweifel olefination with $N$-bromosuccinimide rather than iodine was critical to achieve efficient coupling. Following silyl deprotection, the coupled product $\mathbf{8 0}$ was obtained in $88 \%$ yield as an inconsequential mixture of $Z / E$ isomers. Hydrogenation of the alkene followed by Jones oxidation of the primary alcohol completed the first catalytic enantioselective synthesis of [5]-ladderanoic acid.

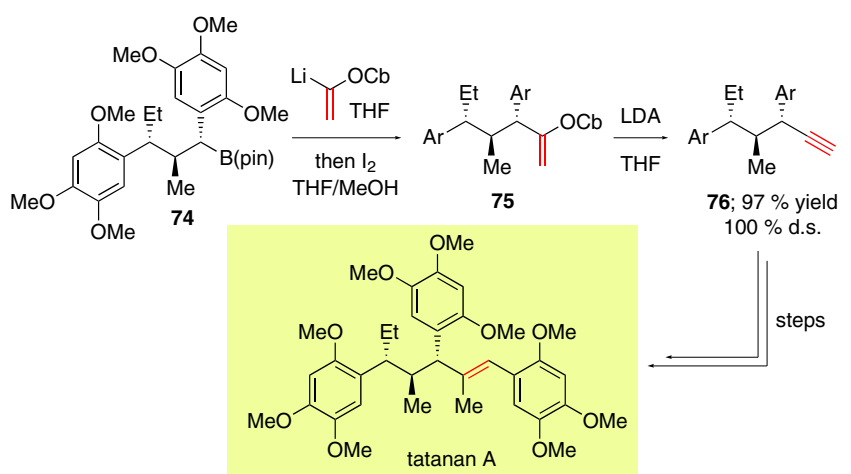

Scheme 28 Total synthesis of tatanan $A ; A r=2,4,5$-trimethoxyphenyl; d.s. = diastereospecifity 

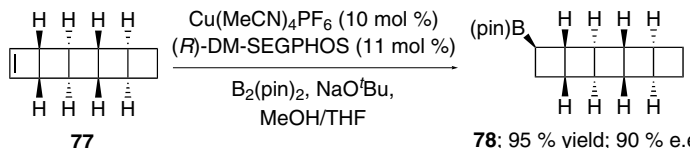

78; $95 \%$ yield; $90 \%$ e.e.

77

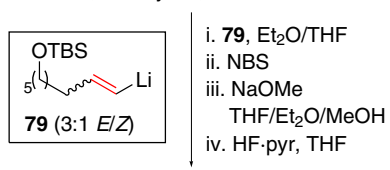

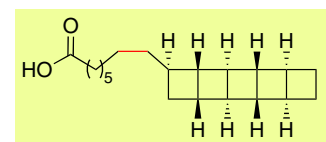

[5]-ladderanoic acid

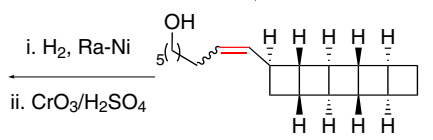

80; $88 \%$ yield
Scheme 29 Zweifel olefination in the synthesis of [5]-ladderanoic acid

Negishi and co-workers have employed a Zweifel olefination in the synthesis of the side chain of (+)-scyphostatin (Scheme 30). ${ }^{42}$ In this case, a boronate complex was formed between vinyl boronic ester $\mathbf{8 1}$ (prepared in 7 steps from allyl alcohol) and methyllithium. After addition of iodine and $\mathrm{NaOH}$ followed by silyl deprotection, trisubstituted alkene $\mathbf{8 2}$ was obtained in $76 \%$ yield. The very high stereoselectivity obtained in this reaction $(>98: 2 \mathrm{E} / \mathrm{Z})$ is particularly noteworthy and represents a significant improvement upon previous synthetic approaches toward this fragment.

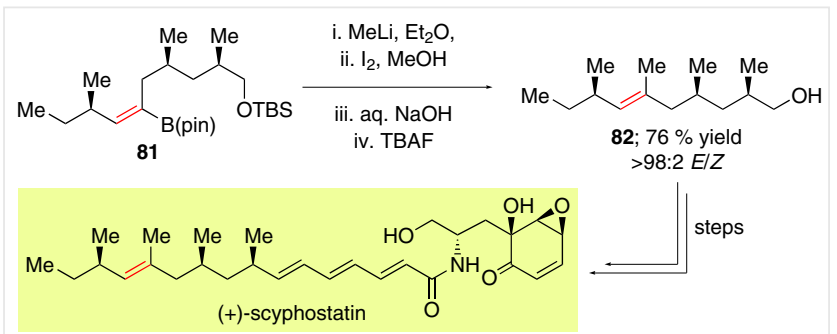

Scheme 30 Construction of the side chain of $(+)$-scyphostatin
Hoveyda and co-workers have employed a similar strategy to synthesize the antitumor agent herboxidiene (Scheme 31). ${ }^{43}$ In this case, Z-vinyl boronic ester $\mathbf{8 3}$ was prepared as a single stereoisomer by a $\mathrm{Cu}$-catalyzed borylation-allylic substitution reaction. Boronic ester $\mathbf{8 3}$ was then converted into trisubstituted alkene $\mathbf{8 4}$ in a Zweifel olefination with methyllithium. The resulting alkene was obtained as a single $E$-isomer in $70 \%$ yield and could be converted into herboxidiene in five steps.

A stereocontrolled synthesis of (-)-filiformin has been reported by Aggarwal and co-workers involving an intramolecular Zweifel olefination (Scheme 32). ${ }^{32}$ Intermediate 85 (synthesized in high stereoselectivity by lithiationborylation) was converted into cyclic boronate complex $\mathbf{8 6}$ by in situ lithium-halogen exchange. Addition of iodine and methanol brought about the desired ring contraction to provide exocyclic alkene $\mathbf{8 7}$ in $97 \%$ yield. Deprotection of the phenolic ether followed by acid-promoted cyclization and bromination completed the synthesis of (-)-filiformin.

\section{Conclusions and Outlook}

Fifty years have passed since the first report by Zweifel and co-workers on the iodine-mediated olefination of vinyl boranes. Since then, this process has evolved into a robust and practical method for the enantiospecific coupling of boronic esters with vinyl metals. Recent contributions have significantly expanded the generality of the process, enabling the efficient coupling of a wide range of different alkenyl partners and allowing increasingly precise control over the stereochemical outcome of the transformation. Rapid progress in enantioselective boronic ester synthesis combined with the extensive applications of chiral alkenes bode well for the continued development and application of the Zweifel olefination in synthesis.

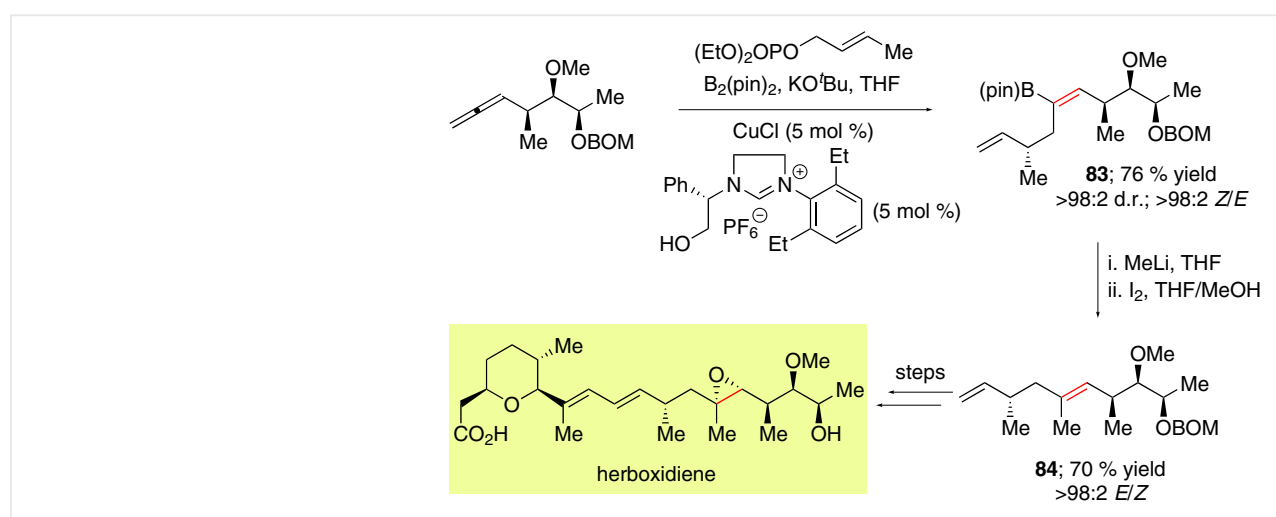

Scheme 31 Total synthesis of herboxidiene; BOM = benzyloxymethyl 


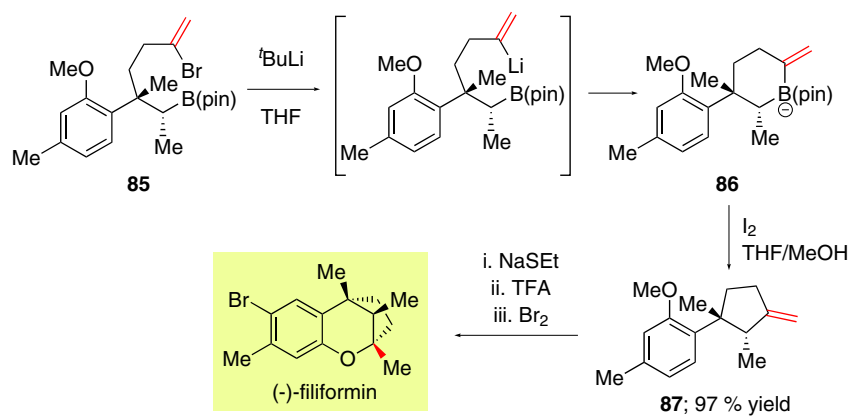

Scheme 32 Synthesis of (-)-filiformin via an intramolecular Zweifel olefination

\section{Funding Information}

We thank EPSRC (EP/I038071/1) and the European Research Council (advanced grant 670668) for financial support.

\section{Acknowledgment}

We are grateful to Dr Eddie Myers and Dr Adam Noble for helpful discussions and suggestions during the preparation of this manuscript.

\section{References}

(1) (a) Stereoselective Alkene Synthesis, In Topics in Current Chemistry,; Wang, J., Ed.; Springer: Heidelberg, 2012. (b) Williams, J. M. J. Preparation of Alkenes: A Practical Approach; Oxford University Press: Oxford, 1996. (c) Negishi, E.; Huang, Z.; Wang, G.; Mohan, S.; Wang, C.; Hattori, H. Acc. Chem. Res. 2008, 41, 1474.

(2) (a) Miyaura, N.; Suzuki, A. Chem. Rev. 1995, 95, 2457. (b) Chemler, S. R.; Trauner, D.; Danishefsky, S. J. Angew. Chem. Int. Ed. 2001, 40, 4544. (c) Nicolaou, K. C.; Bulger, P. G.; Sarlah, D. Angew. Chem. Int. Ed. 2005, 44, 4442. (d) Jana, R.; Pathak, T. P.; Sigman, M. S. Chem. Rev. 2011, 111, 1417. (e) Suzuki, A. Angew. Chem. Int. Ed. 2011, 50, 6722. (f) Li, J.; Ballmer, S. G.; Gillis, E. P.; Fujii, S.; Schmidt, M. J.; Palazzolo, A. M. E.; Lehmann, J. W.; Morehouse, G. F.; Burke, M. D. Science 2015, 347, 1221. (g) Thomas, A. A.; Denmark, S. E. Science 2016, 352, 329.

(3) (a) Leonori, D.; Aggarwal, V. K. Angew. Chem. Int. Ed. 2015, 54, 1082. (b) Wang, C.-Y.; Derosa, J.; Biscoe, M. R. Chem. Sci. 2015, 6, 5105. (c) Cherney, A. H.; Kadunce, N. T.; Reisman, S. E. Chem. Rev. 2015, 115, 9587. (d) Lee, J. C. H.; McDonald, R.; Hall, D. G. Nat. Chem. 2011, 3, 894.

(4) Sun, C.-L.; Shi, Z.-J. Chem. Rev. 2014, 114, 9219.

(5) For other review articles which discuss Zweifel olefination, see: (a) Matteson, D. S. Chem. Rev. 1989, 89, 1535. (b) Matteson, D. S. J. Organomet. Chem. 1999, 581, 51. (c) Scott, H. K.; Aggarwal, V. K. Chem. Eur. J. 2011, 17, 13124. (d) Leonori, D.; Aggarwal, V. K. Acc. Chem. Res. 2014, 47, 3174. (e) Sandford, C.; Aggarwal, V. K. Chem. Commun. 2017, 53, 5481.

(6) Zweifel, G.; Arzoumanian, H.; Whitney, C. C. J. Am. Chem. Soc. 1967, 89, 3652.

(7) Zweifel, G.; Fisher, R. P.; Snow, J. T.; Whitney, C. C. J. Am. Chem. Soc. 1971, 93, 6309.

(8) Matteson, D. S.; Liedtke, J. D. J. Am. Chem. Soc. 1965, 87, 1526.

(9) For the synthesis of trisubstituted alkenes, see: Brown, H. C.; Basavaiah, D.; Kulkarni, S. U. J. Org. Chem. 1982, 47, 171.
(10) Zweifel, G.; Fisher, R. P.; Snow, J. T.; Whitney, C. C. J. Am. Chem. Soc. 1972, 94, 6560 .

(11) LaLima, N. J.; Levy, A. B. J. Org. Chem. 1978, 43, 1279.

(12) Suzuki, A.; Miyaura, N.; Abiko, S.; Itoh, M.; Brown, H. C.; Sinclair, J. A.; Midland, M. M. J. Am. Chem. Soc. 1973, 95, 3080.

(13) For selected examples of alkynylation of boranes and borinic esters, see: (a) Negishi, E.; Lew, G.; Yoshida, T. J. Chem. Soc., Chem. Commun. 1973, 22, 874. (b) Suzuki, A.; Miyaura, N.; Abiko, S.; Itoh, M.; Midland, M. M.; Sinclair, J. A.; Brown, H. C. J. Org. Chem. 1986, 51, 4507. (c) Naruse, M.; Utimoto, K.; Nozaki, H. Tetrahedron 1974, 30, 2159. (d) Naruse, M.; Utimoto, K.; Nozaki, H. Tetrahedron Lett. 1973, 14, 2741. (e) Pelter, A.; Drake, R. A. Tetrahedron Lett. 1988, 29, 4181. (f) Sikorski, J. A.; Bhat, N. G.; Cole, T. E.; Wang, K. K.; Brown, H. C. J. Org. Chem. 1986, 51, 4521. (g) Canterbury, D. P.; Micalizio, G. C. J. Am. Chem. Soc. 2010, 132, 7602.

(14) (a) Aggarwal, V. K.; Fang, G. Y.; Ginesta, X.; Howells, D. M.; Zaja, M. Pure Appl. Chem. 2006, 78, 215. (b) Slayden, S. W. J. Org. Chem. 1981, 46, 2311. (c) Slayden, S. W. J. Org. Chem. 1982, 47, 2753.

(15) Zweifel, G.; Polston, N. L.; Whitney, C. C. J. Am. Chem. Soc. 1968, 90, 6243.

(16) (a) Tripathy, P. B.; Matteson, D. S. Synthesis 1990, 200. (b) Elliott, M. C.; Smith, K.; Jones, D. H.; Hussain, A.; Saleh, B. A. J. Org. Chem. 2013, 78, 3057.

(17) (a) Brown, H. C.; Basavaiah, D. J. Org. Chem. 1982, 47, 3806. (b) Brown, H. C.; Basavaiah, D. J. Org. Chem. 1982, 47, 5407. (c) Brown, H. C.; Basavaiah, D.; Kulkarni, S. U.; Bhat, N. G.; Prasad, J. V. N. V. J. Org. Chem. 1988, 53, 239.

(18) (a) For a review see: Collins, B. S. L.; Wilson, C. M.; Myers, E. L.; Aggarwal, V. K. Angew. Chem. Int. Ed. 2017, in press; DOI: 10.1002/anie.201701963. For selected recent examples, see: (b) Schmidt, J.; Choi, J.; Liu, A. T.; Slusarczyk, M.; Fu, G. C. Science 2016, 354, 1265. (c) Zhang, L.; Lovinger, G. J.; Edelstein, E. K.; Szymaniak, A. A.; Chierchia, M. P.; Morken, J. P. Science 2016, 351, 70. (d) Li, C.; Wang, J.; Barton, L. M.; Yu, S.; Tian, M.; Peters, D. S.; Kumar, M.; Yu, A. W.; Johnson, K. A.; Chatterjee, A. K.; Yan, M.; Baran, P. S. Science 2017, in press; DOI: 10.1126/science.aam7355.

(19) (a) Matteson, D. S.; Jesthi, P. K. J. Organomet. Chem. 1976, 110, 25. (b) Matteson had previously described this work in a review article: Matteson, D. S. Synthesis 1975, 147.

(20) (a) Evans, D. A.; Thomas, R. C.; Walker, J. A. Tetrahedron Lett. 1976, 17, 1427. (b) Evans, D. A.; Crawford, T. C.; Thomas, R. C.; Walker, J. A. J. Org. Chem. 1976, 41, 3947.

(21) Brown, H. C.; Bhat, N. G. J. Org. Chem. 1988, 53, 6009. 
(22) For selected applications of Zweifel reactions of boranes and borinic esters, see: (a) Abatjoglou, A. G.; Portoghese, P. S. Tetrahedron Lett. 1976, 17, 1457. (b) Kulkarni, U. U.; Basavaiah, D.; Brown, H. C. J. Organomet. Chem. 1982, 225, C1. (c) Basavaiah, D.; Brown, H. C. J. Org. Chem. 1982, 47, 1792. (d) Mikhailov, B. M.; Gurskii, M. E.; Pershin, D. G. J. Organomet. Chem. 1983, 246, 19. (e) Hyuga, S.; Takinami, S.; Hara, S.; Suzuki, A. Tetrahedron Lett. 1986, 27, 977. (f) Benmaarouf-Khallaayoun, Z.; Baboulene, M.; Speziale, V.; Lattes, A. J. Organomet. Chem. 1986, 306, 283. (g) Wang, K. K.; Dhumrongvaraporn, S. Tetrahedron Lett. 1987, 28, 1007. (h) Ichikawa, J.; Sonoda, T.; Kobayashi, H. Tetrahedron Lett. 1989, 30, 6379. (i) Hoshi, M.; Masuda, Y.; Arase, A. J. Chem. Soc., Perkin Trans. 1 1990, 12, 3237. (j) Brown, H. C.; Iyer, R. R.; Mahindroo, V. K.; Bhat, N. G. Tetrahedron: Asymmetry 1991, 2, 277. (k) Brown, H. C.; Mandal, A. K. J. Org. Chem. 1992, 57, 4970. (l) Periasamy, M.; Bhanu Prasad, A.; Suseela, Y. Tetrahedron 1995, 51, 2743. (m) Yang, D. Y.; Huang, X. J. Organomet. Chem. 1996, 523, 139. (n) Hoshi, M.; Tanaka, H.; Shirakawa, K.; Arase, A. Chem. Commun. 1999, 627. (o) Smith, K.; Balakit, A. A.; ElHiti, G. A. Tetrahedron 2012, 68, 7834.

(23) Dutheuil, G.; Webster, M. P.; Worthington, P. A.; Aggarwal, V. K. Angew. Chem. Int. Ed. 2009, 48, 6317.

(24) Pulis, A. P.; Blair, D. J.; Torres, E.; Aggarwal, V. K. J. Am. Chem. Soc. 2013, 135, 16054.

(25) (a) Sonawane, R. P.; Jheengut, V.; Rabalakos, C.; LaroucheGauthier, R.; Scott, H. K.; Aggarwal, V. K. Angew. Chem. Int. Ed. 2011, 50, 3760. (b) Shimizu, M. Angew. Chem. Int. Ed. 2011, 50, 5998.

(26) Blair, D. J.; Tanini, D.; Bateman, J. M.; Scott, H. K.; Myers, E. L.; Aggarwal, V. K. Chem. Sci. 2017, 8, 2898.

(27) Linstrumelle, G.; Alami, M. Vinylmagnesium Bromide, In e-EROS Encyclopedia of Reagents for Organic Synthesis; John Wiley \& Sons: Chichester, 2001.

(28) Armstrong, R. J.; Niwetmarin, W.; Aggarwal, V. K. Org. Lett. 2017, 19, 2762.

(29) For related use of DMSO to promote boronate complex formation with vinyl Grignard reagents, see: (a) Lovinger, G. J.; Aparece, M. D.; Morken, J. P. J. Am. Chem. Soc. 2017, 139, 3153. (b) Edelstein, E. K.; Namirembe, S.; Morken, J. P. J. Am. Chem. Soc. 2017, 139, 5027.
(30) For a related example involving trialkylboranes, see: Levy, A. B.; Schwartz, S. J.; Wilson, N.; Christie, B. J. Organomet. Chem. 1978, $156,123$.

(31) Wang, Y.; Noble, A.; Myers, E. L.; Aggarwal, V. K. Angew. Chem. Int. Ed. 2016, 55, 4270.

(32) Blair, D. J.; Fletcher, C. J.; Wheelhouse, K. M. P.; Aggarwal, V. K. Angew. Chem. Int. Ed. 2014, 53, 5552.

(33) (a) Aggarwal, V. K.; Binanzer, M.; de Ceglie, M. C.; Gallanti, M.; Glasspoole, B. W.; Kendrick, S. J. F.; Sonawane, R. P.; VázquezRomero, A.; Webster, M. P. Org. Lett. 2011, 13, 1490. For related examples see: (b) Bhat, N. G.; Lai, W. C.; Carroll, M. B. Tetrahedron Lett. 2007, 48, 4267. (c) Meng, F.; Jang, H.; Hoveyda, A. H. Chem. Eur. J. 2013, 19, 3204.

(34) Armstrong, R. J.; García-Ruiz, C.; Myers, E. L.; Aggarwal, V. K. Angew. Chem. Int. Ed. 2017, 56, 786.

(35) Armstrong, R. J.; Sandford, C.; García-Ruiz, C.; Aggarwal, V. K. Chem. Commun. 2017, 53, 4922.

(36) For other examples of Zweifel olefination in synthesis, see: (a) Man, H.-W.; Hiscox, W. C.; Matteson, D. S. Org. Lett. 1999, 1, 379. (b) Fletcher, C. J.; Blair, D. J.; Wheelhouse, K. M. P.; Aggarwal, V. K. Tetrahedron 2012, 68, 7598. (c) Shoba, V. M.; Thacker, N. C.; Bochat, A. J.; Takacs, J. M. Angew. Chem. Int. Ed. 2016, 55, 1465. (d) Casoni, G.; Myers, E. L.; Aggarwal, V. K. Synthesis 2016, 48, 3241. (e) Chakrabarty, S.; Takacs, J. M. J. Am. Chem. Soc. 2017, 139, 6066.

(37) Varela, A.; Garve, L. K. B.; Leonori, D.; Aggarwal, V. K. Angew. Chem. Int. Ed. 2017, 56, 2127.

(38) Kleinnijenhuis, R. A.; Timmer, B. J. J.; Lutteke, G.; Smits, J. M. M.; de Gelder, R.; van Maarseveen, J. H.; Hiemstra, H. Chem. Eur. J. 2016, 22, 1266.

(39) Blaisdell, T. P.; Morken, J. P. J. Am. Chem. Soc. 2015, 137, 8712.

(40) Noble, A.; Roesner, S.; Aggarwal, V. K. Angew. Chem. Int. Ed. 2016, 55, 15920.

(41) Mercer, J. A. M.; Cohen, C. M.; Shuken, S. R.; Wagner, A. M.; Smith, M. W.; Moss, F. R.; Smith, M. D.; Vahala, R.; GonzalezMartinez, A.; Boxer, S. G.; Burns, N. Z. J. Am. Chem. Soc. 2016, $138,15845$.

(42) Xu, S.; Lee, C.-T.; Rao, H.; Negishi, E. Adv. Synth. Catal. 2011, 353, 2981.

(43) Meng, F.; McGrath, K. P.; Hoveyda, A. H. Nature 2014, 513, 367. 